\title{
Recursions and divisibility properties for combinatorial Macdonald polynomials
}

\author{
Nicholas A. Loehnt and Elizabeth Niese $\|^{*}$ \\ Virginia Tech Dept. of Mathematics \\ received $28^{\text {th }}$ December 2009, revised $12^{\text {th }}$ January 2011, accepted $29^{\text {th }}$ January 2011.
}

For each integer partition $\mu$, let $\widetilde{F}_{\mu}(q, t)$ be the coefficient of $x_{1} \cdots x_{n}$ in the modified Macdonald polynomial $\widetilde{H}_{\mu}$. The polynomial $\widetilde{F}_{\mu}(q, t)$ can be regarded as the Hilbert series of a certain doubly-graded $S_{n}$-module $M_{\mu}$, or as a $q, t$-analogue of $n$ ! based on permutation statistics $\operatorname{inv}_{\mu}$ and $\operatorname{maj}_{\mu}$ that generalize the classical inversion and major index statistics. This paper uses the combinatorial definition of $\widetilde{F}_{\mu}$ to prove some recursions characterizing these polynomials, and other related ones, when $\mu$ is a two-column shape. Our result provides a complement to recent work of Garsia and Haglund, who proved a different recursion for two-column shapes by representation-theoretical methods. For all $\mu$, we show that $\widetilde{F}_{\mu}(q, t)$ is divisible by certain $q$-factorials and $t$-factorials depending on $\mu$. We use our recursion and related tools to explain some of these factors bijectively. Finally, we present fermionic formulas that express $\widetilde{F}_{\left(2^{n}\right)}(q, t)$ as a sum of $q, t$-analogues of $n ! 2^{n}$ indexed by perfect matchings.

Keywords: Macdonald polynomials, Garsia-Haiman modules, Hilbert series, bijections, recursions, $q$-analogues

\section{Introduction}

The Macdonald polynomials $P_{\mu}[$ Mac88] play a prominent role in algebraic combinatorics and the theory of symmetric functions. The polynomials $P_{\mu}$, as $\mu$ ranges over all integer partitions of $n$, form a basis for the vector space of symmetric polynomials of degree $n$ with coefficients in the field $\mathbb{Q}(q, t)$. By specializing the parameters $q$ and $t$ in various ways, one can obtain many classical symmetric function bases from the Macdonald basis. For instance, $\left.P_{\mu}\right|_{t=1}$ is the monomial symmetric polynomial $m_{\mu} ;\left.P_{\mu}\right|_{q=1}$ is the elementary symmetric polynomial $e_{\mu^{\prime}} ;\left.P_{\mu}\right|_{q=t}$ is the Schur symmetric polynomial $s_{\mu}$; and $\left.P_{\mu}\right|_{q=0}$ is a Hall-Littlewood polynomial [Mac95].

Garsia, Haglund, and Haiman (among others) studied modified versions of Macdonald polynomials, denoted $\widetilde{H}_{\mu}$, that have remarkable connections to representation theory and combinatorics [GH93, Hag04, HHL05a]. To compute the modified polynomials $\widetilde{H}_{\mu}$ from the original polynomials $P_{\mu}$, one first multiplies each $P_{\mu}$ by a certain scalar in $\mathbb{Q}(q, t)$ to obtain the integral forms $J_{\mu}$, then applies a suitable

\footnotetext{
${ }^{\dagger}$ Email: nloehr@vt.edu.

‡Email: eniese@vt.edu.

1365-8050 @ 2011 Discrete Mathematics and Theoretical Computer Science (DMTCS), Nancy, France
} 
plethystic transform to obtain $H_{\mu}$, and finally sets $\widetilde{H}_{\mu}=\left.t^{n(\mu)} H_{\mu}\right|_{t \rightarrow 1 / t}$. See [GH98, HHL05b] for more details. One can also give a direct characterization of $\widetilde{H}_{\mu}$ in terms of triangularity and normalization axioms [HHL05a, HHL05b], or one can define $\widetilde{H}_{\mu}$ to be the eigenfunctions of the linear operator $\Delta_{1}[\mathrm{GH} 96]$.

In [GH93], Garsia and Haiman defined doubly-graded $S_{n}$-modules $M_{\mu}$ and conjectured that the Frobenius series for these modules were none other than the modified Macdonald polynomials $\widetilde{H}_{\mu}$. This conjecture was ultimately proved by Haiman using deep results from commutative algebra and algebraic geometry [Hai01, Hai02]. Let $\widetilde{F}_{\mu} \in \mathbb{Q}(q, t)$ be the coefficient of $x_{1} x_{2} \cdots x_{n}$ in $\widetilde{H}_{\mu}$; equivalently, $\widetilde{F}_{\mu}=\left\langle\widetilde{H}_{\mu}, h_{\left(1^{n}\right)}\right\rangle$. It follows from Haiman's result that $\widetilde{F}_{\mu}$ is the Hilbert series of the doubly-graded module $M_{\mu}$; i.e.,

$$
\widetilde{F}_{\mu}(q, t)=\sum_{i \geq 0} \sum_{j \geq 0} \operatorname{dim}\left(M_{\mu}^{(i, j)}\right) q^{i} t^{j}
$$

where $M_{\mu}^{(i, j)}$ denotes the bihomogeneous component of $M_{\mu}$ of bidegree $(i, j)$.

In a groundbreaking paper [Hag04], Haglund conjectured a combinatorial formula for $\widetilde{F}_{\mu}$ of the form

$$
\widetilde{F}_{\mu}=\sum_{w \in S_{n}} q^{\operatorname{inv}_{\mu}(w)} t^{\operatorname{maj}_{\mu}(w)},
$$

where $\operatorname{inv}_{\mu}, \operatorname{maj}_{\mu}: S_{n} \rightarrow \mathbb{N}$ are suitable permutation statistics parametrized by $\mu$. This formula leads directly to a combinatorial prescription for the expansion of $\widetilde{H}_{\mu}$ into fundamental quasisymmetric polynomials. These formulas, which are described in more detail in $\$ 2$ below, were proved by Haglund, Haiman, and Loehr [HHL05a, HHL05b]. Haglund's formula opened the way to new explorations of the combinatorial properties of Macdonald polynomials and the associated Hilbert series $\widetilde{F}_{\mu}$.

A problem of ongoing interest involves the search for recursions characterizing the polynomials $\widetilde{F}_{\mu}$, as $\mu$ ranges over all integer partitions. One such recursion follows from a version of the Pieri rules for Macdonald polynomials [Mac95]. This recursion has the form

$$
\widetilde{F}_{\mu}(q, t)=\sum_{\nu \rightarrow \mu} c_{\mu, \nu}(q, t) \widetilde{F}_{\nu}(q, t), \quad \widetilde{F}_{(0)}(q, t)=1,
$$

where we sum over all partitions $\nu$ that can be obtained from $\mu$ by removing a single corner box, and $c_{\mu, \nu}(q, t) \in \mathbb{Q}(q, t)$ is a complicated quotient of two polynomials built from the arm and leg numbers of the cells in $\mu$ and $\nu$ [GH98]. While this recursion is very striking, the presence of rational coefficients makes it difficult to use in combinatorial settings. For instance, there is presently no known combinatorial proof of this recursion based on (1), nor can one easily deduce the polynomiality of $\widetilde{F}_{\mu}$ from (2).

In a very recent paper [GHar], Garsia and Haglund found a new recursion valid for polynomials $\widetilde{F}_{\mu}$ indexed by shapes $\mu$ with two columns. They proved this recursion using a multitude of clever representation-theoretical manipulations. In related work, M. Yoo used (1) to derive a combinatorial formula for polynomials $\widetilde{F}_{\mu}$ indexed by partitions $\mu$ of hook shape [Yoo09]. Some results for two-row shapes and rectangles appear in J. Bandlow's doctoral dissertation [Ban07].

The Garsia-Haglund recursion builds up a given shape $\mu$ from smaller shapes $\nu$ obtained by removing one of the inner corners of $\mu$. In this paper, we shall present different recursions characterizing the polynomials $\widetilde{F}_{\mu}$ (where $\mu$ has two columns) that are based on removing the bottom row of $\mu$. Our recursions are stated in Theorem 6.10 and Theorem 6.16 below. We will give a fully combinatorial proof of 
our recursions using bijections and combinatorial operations on permutations weighted by the statistics $\operatorname{inv}_{\mu}$ and $\operatorname{maj}_{\mu}$. For our approach, it is more convenient to use $F_{\mu}(q, t)=t^{n(\mu)} \widetilde{F}_{\mu}(q, 1 / t)$; this entails replacing maj $_{\mu}$ by a related statistic $\operatorname{comaj}_{\mu}$, which is defined in 82 .

For $n \in \mathbb{N}$, let $[n]_{t}=1+t+t^{2}+\cdots+t^{n-1}$ and $[n] !_{t}=\prod_{i=1}^{n}[i]_{t}$. It is straightforward to deduce from Haglund's combinatorial formula (1) that $F_{\left(1^{n}\right)}(q, t)=\widetilde{F}_{\left(1^{n}\right)}(q, t)=[n] !_{t}$ and $F_{(n)}(q, t)=\widetilde{F}_{(n)}(q, t)=$ $[n] !_{q}$. One consequence of our recursion is a combinatorial explanation of the fact that $F_{\left(2^{m}\right)}(q, t)$ is divisible by $[m] !_{t}$. We will prove the following more general divisibility property in $\$ 4$

Theorem 1.1 Let $\mu=\left(1^{n_{1}} 2^{n_{2}} \cdots k^{n_{k}}\right)$ be an integer partition with $\mu^{\prime}=\left(1^{m_{1}} 2^{m_{2}} \cdots p^{m_{p}}\right)$.

(a) $\widetilde{F}_{\mu}(q, t)$ and $F_{\mu}(q, t)$ are divisible by $\left[m_{1}\right] !_{q}\left[m_{2}\right] !_{q} \cdots\left[m_{p}\right] !_{q}$.

(b) $\widetilde{F}_{\mu}(q, t)$ and $F_{\mu}(q, t)$ are divisible by $\left[n_{1}\right] !_{t}\left[n_{2}\right] !_{t} \cdots\left[n_{k}\right] !_{t}$.

As we will see, (a) follows bijectively from a certain combinatorial move on permutations. Then (b) follows algebraically from (a) using the known symmetry property $\widetilde{H}_{\mu}(X ; q, t)=\widetilde{H}_{\mu^{\prime}}(X ; t, q)$ of Macdonald polynomials [Mac95]. One of our motivations for developing the recursions below is to obtain a greater understanding of why (b) holds at the combinatorial level. Proving (b) bijectively for general $\mu$ appears to be a difficult problem. We hope that the special cases considered here will yield some clues to the even more challenging problem of giving a bijective proof of the general symmetry property $\widetilde{F}_{\mu}(q, t)=\widetilde{F}_{\mu^{\prime}}(t, q)$.

\section{Combinatorial Formula for $F_{\mu}$}

This section reviews Haglund's combinatorial formula for $\widetilde{F}_{\mu}(q, t)$ and defines a related polynomial $F_{\mu}(q, t)$.

Definition 2.1 A partition of a positive integer $n$ is a sequence $\mu=\left(\mu_{1}, \mu_{2}, \ldots, \mu_{k}\right)$ with $\mu_{1}+\mu_{2}+\cdots+$ $\mu_{k}=n$ and $\mu_{1} \geq \mu_{2} \geq \cdots \geq \mu_{k}>0$, for some $k$. Let $\operatorname{Par}(n)$ be the set of all partitions of $n$.

Definition 2.2 A standard filling $T$ of shape $\mu \in \operatorname{Par}(n)$ is a placement of the integers $1,2, \ldots, n$ in the Ferrers diagram of $\mu$ with each integer used exactly once. A filling can be identified with a permutation $w \in S_{n}$ by reading the rows of the diagram from left to right, beginning with the shortest row. The column words of a filling are obtained by reading the columns from top to bottom.

Example 2.3 For $\mu=(5,3,1)$, an example of a standard filling is:

$$
T=\begin{array}{|l|l|l|l|l|}
\hline 4 & \multicolumn{4}{|l}{} \\
\hline 3 & 1 & 6 & \multicolumn{2}{|c}{} \\
\hline 2 & 8 & 5 & 9 & 7 \\
\hline
\end{array}
$$

This filling is identified with the permutation $w=431628597 \in S_{9}$. The column words for this filling are $432,18,65,9$, and 7 .

In order to define the statistics $\operatorname{inv}_{\mu}(T)$ and $\operatorname{comaj}_{\mu}(T)$, we first recall some classical permutation statistics defined for $w \in S_{n}$. 
Definition 2.4 Fix $w=w_{1} w_{2} \cdots w_{n} \in S_{n}$. An inversion of $w$ is a pair $i<j$ such that $w_{i}>w_{j}$. Note that $i$ and $j$ do not need to be consecutive. Let $\operatorname{inv}(w)$ be the number of inversions of $w$. A descent of $w$ is an index $i<n$ with $w_{i}>w_{i+1}$. The descent set of $w$ is $\operatorname{Des}(w)=\left\{i: w_{i}>w_{i+1}\right\}$. The major index of $w$ is $\operatorname{maj}(w)=\sum_{i \in \operatorname{Des}(w)} i$. Similarly, define the ascent $\operatorname{set} \operatorname{Asc}(w)=\left\{i: w_{i}<w_{i+1}\right\}$ and the co-major index $\operatorname{comaj}(w)=\sum_{i \in \operatorname{Asc}(w)} i$.

Example 2.5 For $w=52341 \in S_{5}, \operatorname{inv}(w)=7, \operatorname{Des}(w)=\{1,4\}, \operatorname{maj}(w)=5, \operatorname{Asc}(w)=\{2,3\}$, and $\operatorname{comaj}(w)=5$.

Definition 2.6 For a standard filling $T$ of $\mu \in \operatorname{Par}(n)$, we define a triple to be three cells in $T$ with entries $a, b, c$, arranged as shown here:

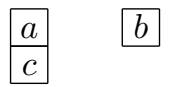

where $a$ is directly above $c$, and $b$ is in the same row as a and to the right of $a$. If the cells containing $a$ and $b$ are in the lowest row, take $c=\infty$. This triple is an inversion triple of T iff $a<c<b$ or $c<b<a$ or $b<a<c$. We define

$$
\operatorname{inv}_{\mu}(T)=\text { number of inversion triples of } T \text {. }
$$

Definition 2.7 For a standard filling $T$ of $\mu \in \operatorname{Par}(n)$, the $\mu$-major index of $T$ is

$$
\operatorname{maj}_{\mu}(T)=\text { sum of the major indices of the column words of } T \text {. }
$$

Definition 2.8 For a partition $\mu=\left(\mu_{1}, \mu_{2}, \ldots, \mu_{k}\right) \in \operatorname{Par}(n)$, define $n(\mu)=\sum_{i=1}^{k}(i-1) \mu_{i}$.

Example 2.9 For the filling $T$ of $\mu=(3,3,2,1)$ given by

$$
T=\begin{array}{l|l|l|}
\hline 6 & & \\
\hline 2 & 5 & \\
\hline 1 & 3 & 8 \\
\hline 7 & 4 & 9 \\
\hline
\end{array},
$$

$$
\operatorname{maj}_{\mu}(T)=\operatorname{maj}(6217)+\operatorname{maj}(534)+\operatorname{maj}(89)=3+1+0=4,
$$

$n(\mu)=10$, and $\operatorname{inv}_{\mu}(T)=3$ since the inversion triples are $4<7<\infty, 1<7<8$, and $3<4<8$.

Definition 2.10 For all partitions $\mu$, let $\mathcal{F}_{\mu}$ be the set of all standard fillings of the shape $\mu$.

Haglund's combinatorial formula [Hag04] for $\widetilde{F}_{\mu}(q, t)$ is

$$
\widetilde{F}_{\mu}(q, t)=\sum_{T \in \mathcal{F}_{\mu}} q^{\operatorname{inv}_{\mu}(T)} t^{\operatorname{maj}_{\mu}(T)} .
$$

Definition 2.11 For $T \in \mathcal{F}_{\mu}$, define $\operatorname{comaj}_{\mu}(T)=n(\mu)-\operatorname{maj}_{\mu}(T)$. Note that $\operatorname{comaj}_{\mu}(T)$ can also be written as the sum of the co-major indices of the column words of $T$.

In the following, we will use the specialization

$$
F_{\mu}(q, t)=t^{n(\mu)} \widetilde{F}_{\mu}(q, 1 / t)=\sum_{T \in \mathcal{F}_{\mu}} q^{\operatorname{inv}_{\mu}(T)} t^{\operatorname{comaj}_{\mu}(T)} .
$$

$F_{\mu}(q, t)$ is the coefficient of $x_{1} x_{2} \cdots x_{n}$ in the (unmodified) Macdonald polynomial $H_{\mu}\left(x_{1}, x_{2}, \ldots, x_{n} ; q, t\right)$. 


\section{The Inversion Flip Operation}

This section describes a combinatorial operation on fillings called the inversion flip that will allow us to partially sort the bottom row of a filling $T \in \mathcal{F}_{\mu}$. A similar operation on fillings that may have repeated symbols was studied by Bandlow [Ban07, Section 5.5].

Definition 3.1 Suppose $\mu \in \operatorname{Par}(n)$ and $i \in \mathbb{N}^{+}$satisfy $\mu_{i}^{\prime}=\mu_{i+1}^{\prime}$. Define the inversion flip move $s_{i}: \mathcal{F}_{\mu} \rightarrow \mathcal{F}_{\mu}$ as follows:

- Given $T \in \mathcal{F}_{\mu}$, let a (resp. b) be the entry of $T$ at the bottom of column $i$ (resp. $\left.i+1\right)$.

- Switch entries a and $b$ in the bottom row as shown here:

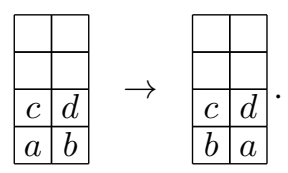

- If $a, c, d$ and $b, c, d$ are either both inversion triples or both not inversion triples, the move is complete. Otherwise, apply $s_{i}$ recursively to the filling $T^{\prime}$ of $\left(\mu_{2}, \mu_{3}, \ldots\right)$ obtained by ignoring the bottom row of $T$.

Lemma 3.2 Given $\mu \in \operatorname{Par}(n)$ and $i, j \in \mathbb{N}^{+}$with $\mu_{i}^{\prime}=\mu_{i+1}^{\prime}$ and $\mu_{j}^{\prime}=\mu_{j+1}^{\prime}$,

(a) $s_{i}^{2}=s_{i} \circ s_{i}=\mathrm{id}_{\mathcal{F}_{\mu}}$;

(b) $s_{i} \circ s_{j}=s_{j} \circ s_{i}$ when $|i-j| \geq 2$.

Proof: Both properties follow directly from the definition of $s_{i}$.

Remark 3.3 The $s_{i}$ 's do not satisfy the braid relations in general; i.e., when $\mu_{i}^{\prime}=\mu_{i+1}^{\prime}=\mu_{i+2}^{\prime}$, we may have $s_{i} \circ s_{i+1} \circ s_{i} \neq s_{i+1} \circ s_{i} \circ s_{i+1}$. This can be seen in Figure 1 .

$$
\begin{aligned}
& \begin{array}{|l|l|l|}
\hline 1 & 3 & 5 \\
\hline 2 & 6 & 4 \\
\hline
\end{array} \stackrel{s_{2}}{\rightarrow} \begin{array}{|l|l|l}
\hline 1 & 5 & 3 \\
\hline 2 & 4 & 6 \\
\hline
\end{array} \stackrel{s_{1}}{\rightarrow} \begin{array}{|l|l|l|}
\hline 1 & 5 & 3 \\
\hline 4 & 2 & 6 \\
\hline
\end{array} \stackrel{s_{2}}{\rightarrow} \begin{array}{|l|l|l|}
\hline 1 & 5 & 3 \\
\hline 4 & 6 & 2 \\
\hline
\end{array} \\
& \begin{array}{|l|l|l|}
\hline 1 & 3 & 5 \\
\hline 2 & 6 & 4 \\
\hline
\end{array} \stackrel{s_{1}}{\rightarrow} \begin{array}{|l|l|l|}
3 & 1 & 5 \\
\hline 6 & 2 & 4 \\
\hline
\end{array} \stackrel{s_{2}}{\rightarrow} \begin{array}{|l|l|l|}
3 & 1 & 5 \\
\hline 6 & 4 & 2 \\
\hline
\end{array} \stackrel{s_{1}}{\rightarrow} \begin{array}{|l|l|l|}
\hline 3 & 1 & 5 \\
\hline 4 & 6 & 2 \\
\hline
\end{array}
\end{aligned}
$$

Fig. 1: $s_{i} \circ s_{i+1} \circ s_{i} \neq s_{i+1} \circ s_{i} \circ s_{i+1}$ in general.

Proposition 3.4 Given $\mu \in \operatorname{Par}(n)$ and $i \in \mathbb{N}^{+}$with $\mu_{i}^{\prime}=\mu_{i+1}^{\prime}$, let $T \in \mathcal{F}_{\mu}$ have entries a and $b$ in the bottom row of columns $i$ and $i+1$, respectively. Then:

(a) $\operatorname{comaj}_{\mu}\left(s_{i}(T)\right)=\operatorname{comaj}_{\mu}(T)$;

(b) $\operatorname{maj}_{\mu}\left(s_{i}(T)\right)=\operatorname{maj}_{\mu}(T)$; 
(c) $\operatorname{inv}_{\mu}\left(s_{i}(T)\right)=\operatorname{inv}_{\mu}(T)+ \begin{cases}1 & \text { if } a<b ; \\ -1 & \text { if } b<a .\end{cases}$

Proof: (cf. [Ban07, Section 5.5]) Since every column contributes independently to comaj ${ }_{\mu}$ and maj $_{\mu}$, to prove (a) and (b) it is sufficient to consider a filling $T$ of shape $\mu=\left(2^{n}\right)$ and $i=1$. By 3.2 (a) we may also assume that the bottom row of $T$ is increasing. We must show that $\operatorname{comaj}_{\mu}\left(s_{1}(T)\right)=\operatorname{comaj}_{\mu}(T)$, which automatically yields $\operatorname{maj}_{\mu}\left(s_{1}(T)\right)=\operatorname{maj}_{\mu}(T)$. This result is true when $n=1$.

When $n=2$, we can write

$$
T=\begin{array}{|l|l|}
\hline c & d \\
\hline a & b \\
\hline
\end{array}
$$

If

$$
s_{1}(T)=\begin{array}{|l|l|}
\hline d & c \\
\hline b & a \\
\hline
\end{array}
$$

column words are preserved, so $\operatorname{comaj}_{\mu}\left(s_{1}(T)\right)=\operatorname{comaj}_{\mu}(T)$ and $\operatorname{maj}_{\mu}\left(s_{1}(T)\right)=\operatorname{maj}_{\mu}(T)$.

It is also possible that

$$
s_{1}(T)=\begin{array}{|l|l|}
c & d \\
\hline b & a \\
\hline
\end{array}
$$

In this case, if $a, c, d$ formed an inversion triple in $T$, then $b, c, d$ forms an inversion triple in $s_{1}(T)$. If $a<b<d<c, d<c<a<b$, or $c<a<b<d$, then the location and number of the ascents are preserved, so comaj ${ }_{\mu}\left(s_{1}(T)\right)=\operatorname{comaj}_{\mu}(T)$. If $a<d<c<b$, the number of ascents is preserved, but the column in which the ascent is located changes. However, since the columns are of equal height, $\operatorname{comaj}_{\mu}\left(s_{1}(T)\right)=\operatorname{comaj}_{\mu}(T)$. Similarly, if $a, c, d$ is not an inversion triple in $T$, then $b, c, d$ is not an inversion triple in $s_{1}(T)$. If $a<b<c<d$, $c<d<a<b$, or $d<a<b<c$, the location and number of ascents are preserved. If $a<c<d<b$, the number of ascents are preserved, but the column in which the ascent is located changes. As before, since the columns are of equal height, $\operatorname{comaj}_{\mu}\left(s_{1}(T)\right)=\operatorname{comaj}_{\mu}(T)$. By induction, this result holds for any number of rows.

To show that $\operatorname{inv}_{\mu}\left(s_{i}(T)\right)=\operatorname{inv}_{\mu}(T)+1$, we must show that the inversion flip does not affect the total number of inversion triples, excluding the triple $a, b, \infty$. By the definition of the inversion flip, it is sufficient to consider triples positioned as shown:

$$
\begin{array}{|l|l|l|}
\hline c & d \\
\hline a & b & z \\
\hline
\end{array}
$$

since all other triples in $T$ will be preserved. Once again, there are two possibilities. First, if

$$
s_{i}(T)=\begin{array}{l|l|l|}
d & c \\
\hline b & a & \ldots \\
\hline
\end{array}
$$

the inversion triples themselves are preserved. On the other hand, if

$$
s_{i}(T)=\begin{array}{|l|l|l|}
c & d \\
\hline b & a
\end{array} \quad \begin{aligned}
& z \\
& \hline
\end{aligned}
$$

to show that the total number of inversion triples is preserved requires a tedious case analysis. We present several cases here and leave the remainder to the reader. First, suppose $z<a<b<c<d$. Then none of $z<a<c, z<b<c, z<a<d$, and $z<b<d$ are inversion triples. Next, if $a<z<b<c<d$, 
then $a<z<c$ is an inversion triple in $T$, and $z<b<c$ is not an inversion triple in $s_{i}(T)$. On the other hand, $z<b<d$ is not an inversion triple in $T$, but $a<z<d$ is an inversion triple in $s_{i}(T)$. Thus, the total number of inversion triples is preserved. The remaining cases are similar.

\section{Divisibility Properties for $F_{\mu}$}

We now prove Theorem 1.1 Given $\mu=\left(1^{n_{1}} 2^{n_{2}} \cdots k^{n_{k}}\right)$ with $\mu^{\prime}=\left(1^{m_{1}} 2^{m_{2}} \cdots p^{m_{p}}\right)$, we must prove that $F_{\mu}(q, t)$ and $\widetilde{F}_{\mu}(q, t)$ are divisible by (a) $\left[m_{1}\right] !_{q}\left[m_{2}\right] !_{q} \cdots\left[m_{p}\right] !_{q}$ and (b) $\left[n_{1}\right] !_{t}\left[n_{2}\right] !_{t} \cdots\left[n_{k}\right] !_{t}$.

First we prove (a) bijectively by using the inversion flip. We begin by considering a filling $T$ of shape $\mu=\left(m^{n}\right)$. For $1 \leq i \leq m$, let $k_{i}$ be the $i$ th smallest entry in the bottom row of $T$, and let $c_{1}, c_{2}, \ldots, c_{m}$ be the cells in the bottom row of $\mu$. If $k_{m}$ is in cell $c_{i}$, applying $s_{m-1} \circ s_{m-2} \circ \cdots \circ s_{i}$ will move $k_{m}$ to cell $c_{m}$. Continue likewise to move each $k_{i}$ into cell $c_{i}$ in decreasing order. The sequence of inversion flips used to sort the $k_{i}$ 's gives rise to a permutation $w \in S_{m}$, by replacing each $s_{j}$ in the sequence by the basic transposition $(j, j+1)$. Let $T^{\prime}$ be the filling that results from sorting $T$ in this way. By 3.4.c), $\operatorname{inv}_{\mu}(T)=\operatorname{inv}_{\mu}\left(T^{\prime}\right)+\operatorname{inv}(w)$ and $\operatorname{comaj}_{\mu}(T)=\operatorname{comaj}_{\mu}\left(T^{\prime}\right)$. Hence,

$$
\begin{aligned}
& F_{\mu}(q, t)=\sum_{T \in \mathcal{F}_{\mu}} q^{\mathrm{inv}_{\mu}(T)} t^{\operatorname{comaj}_{\mu}(T)} \\
& =\sum_{\substack{T^{\prime} \in \mathcal{F}_{\mu} \text { with } \\
\text { increasing row 1 }}} \sum_{w \in S_{m}} q^{\operatorname{inv}_{\mu}\left(T^{\prime}\right)+\operatorname{inv}(w)} t^{\operatorname{comaj}_{\mu}\left(T^{\prime}\right)} \\
& =\left(\sum_{w \in S_{m}} q^{\operatorname{inv}(w)}\right)\left(\sum_{\substack{T^{\prime} \in \mathcal{F}_{\mu} \text { with } \\
\text { increasing row 1 }}} q^{\operatorname{inv}_{\mu}\left(T^{\prime}\right)} t^{\operatorname{comaj}_{\mu}\left(T^{\prime}\right)}\right) \\
& =[m] !_{q}\left(\sum_{\substack{T^{\prime} \in \mathcal{F}_{\mu} \text { with } \\
\text { increasing row } 1}} q^{\operatorname{inv}_{\mu}\left(T^{\prime}\right)} t^{\operatorname{comaj}_{\mu}\left(T^{\prime}\right)}\right) .
\end{aligned}
$$

This result can be extended to $\mu \in \operatorname{Par}(n)$ with $\mu^{\prime}=\left(1^{m_{1}} 2^{m_{2}} \cdots p^{m_{p}}\right)$ by applying the above argument to each set of $m_{i}$ columns of equal height. Thus $F_{\mu}(q, t)$ is divisible by $\left[m_{1}\right] !_{q} \cdots\left[m_{p}\right] !_{q}$. It follows that $\widetilde{F}_{\mu}(q, t)=t^{n(\mu)} F_{\mu}(q, 1 / t)$ is divisible by the same factors. We remark that the divisibility property 1.1 (a) for rectangles is also a consequence of [Ban07], Corollary 3 in Section 5.5.

Part (b) of 1.1 follows algebraically from (a) and the fact that $\widetilde{F}_{\mu}(q, t)=\widetilde{F}_{\mu^{\prime}}(t, q)$.

Example 4.1 For $\mu=(5,5,2,2)$, consider the filling

$$
T=\begin{array}{r|c|c|c|c|}
\hline 4 & 2 & \multicolumn{3}{|c}{} \\
\cline { 1 - 2 } 9 & 12 & \multicolumn{3}{|c}{} \\
\cline { 1 - 2 } 13 & 8 & 5 & 1 & 14 \\
\hline 11 & 3 & 10 & 6 & 7 \\
\hline
\end{array}
$$


Then $s_{1}\left(s_{4}\left(s_{3}(T)\right)\right)=s_{4}\left(s_{3}\left(s_{1}(T)\right)\right)$, and

$$
s_{1}\left(s_{4}\left(s_{3}(T)\right)\right)=\begin{array}{|l|l|l|l|l|l|}
\hline 4 & 2 & & \\
\cline { 1 - 1 } 9 & 12 & & & \\
\hline 8 & 13 & 5 & 1 & 14 \\
\hline 3 & 11 & 6 & 7 & 10 \\
\hline
\end{array} .
$$

Considering the first two columns, the transposition associated with $s_{1}$ is $(1,2)$, so the permutation generated by the inversion flip is $12 \in S_{2}$. Considering the last three columns, the transpositions associated with $s_{3}$ and $s_{4}$ are $(3,4)$ and $(4,5)$, respectively. This gives us the permutation 534 of $\{3,4,5\}$.

\section{The Cyclic Shift Operation}

We wish to obtain a combinatorial explanation for part (b) of Theorem 1.1 at least in the case where $\mu$ is a two-column rectangle. For this, we must first define another bijection on standard fillings.

Definition 5.1 Given $\mu \in \operatorname{Par}(n)$ and $T \in \mathcal{F}_{\mu}$, define the cyclic shift of $T$, denoted $\operatorname{cyc}(T)$, by replacing each entry $c$ in $T$ by $(c+1) \bmod n$. Here we use the convention that a $\bmod n \in\{1,2, \ldots, n\}$.

Lemma 5.2 For all $\mu \in \operatorname{Par}(n)$, $\operatorname{cyc}^{n}=\operatorname{cyc} \circ$ cyc $\circ \cdots \circ$ cyc $=\operatorname{id}_{\mathcal{F}_{\mu}}$.

Proof: Let $T \in \mathcal{F}_{\mu}$. Each entry $c \in T$ will be replaced by the entry $(c+n) \bmod n=c$ in $\operatorname{cyc}^{n}(T)$. Thus $\operatorname{cyc}^{n}(T)=T$.

Proposition 5.3 Let $\mu \in \operatorname{Par}(n)$. For $T \in \mathcal{F}_{\mu}$, where $n$ is not in the bottom row,

(a) $\operatorname{comaj}_{\mu}(\operatorname{cyc}(T))=\operatorname{comaj}_{\mu}(T)+1$;

(b) $\operatorname{maj}_{\mu}(\operatorname{cyc}(T))=\operatorname{maj}_{\mu}(T)-1$;

(c) $\operatorname{inv}_{\mu}(\operatorname{cyc}(T))=\operatorname{inv}_{\mu}(T)$.

Proof: This can be seen by considering what happens to the cell $c$ containing $n$ in a filling.

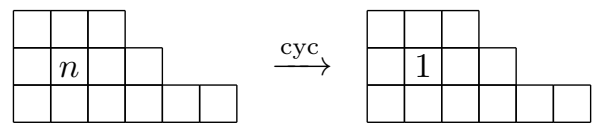

The ascent between $n$ and the cell above (if any) is shifted downward by a unit, while the descent between $n$ and the cell below are shifted up one unit. All other ascents and descents are unaffected. All triples in $T$ which do not involve $n$ are preserved. Triples in $T$ which include $n$ have the form $a<b<n$. The corresponding triple in $T^{\prime}$ will be $1<a+1<b+1$. Thus, the status of the triple will be preserved. The assumption that $n$ is not in the bottom row is needed to ensure that no triple involving $n$ also involves an $\infty$ below the bottom row. 
Example 5.4 For $\mu=(3,3,2)$ and $n=8$,

$$
\begin{aligned}
& T=\begin{array}{l|l|l}
\hline 3 & 8 & \\
\hline 5 & 2 & 1 \\
\hline 4 & 7 & 6 \\
\hline
\end{array} \quad \text { and } \quad \operatorname{cyc}(T)=\begin{array}{|l|l|l|}
\hline 4 & 1 & \\
6 & 3 & 2 \\
\hline 5 & 8 & 7 \\
\hline
\end{array} \text {, } \\
& \text { the statistics are: } \\
& \operatorname{inv}_{\mu}(T)=3 ; \quad \operatorname{inv}_{\mu}(\operatorname{cyc}(T))=3 ; \\
& \operatorname{comaj}_{\mu}(T)=4 ; \quad \operatorname{comaj}_{\mu}(\operatorname{cyc}(T))=5 \text {; } \\
& \operatorname{maj}_{\mu}(T)=3 ; \quad \operatorname{maj}_{\mu}(\operatorname{cyc}(T))=2 \text {. }
\end{aligned}
$$

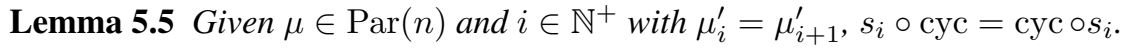

Proof: Since $s_{i}$ only modifies two adjacent columns in the partition, we are reduced to considering $T \in \mathcal{F}_{\left(2^{n}\right)}$. We argue by induction on $n$. If $2 n$ is not in the bottom row of $T$, then, since cyc preserves both the number and location of inversion triples, $\left(s_{1} \circ \mathrm{cyc}\right)(T)=\left(\operatorname{cyc}_{0} \circ s_{1}\right)(T)$ follows by the recursive definition of $s_{1}$ and induction.

If $2 n$ is in the bottom row of $T$, we can assume the bottom row is increasing by 3.2 (a). Then

$$
T=\begin{array}{|c|c|c|}
\hline \vdots & \vdots \\
\hline c & d \\
\hline a & 2 n \\
\hline
\end{array} \quad \text { and } \quad \operatorname{cyc}(T)=\begin{array}{|c|c|}
\hline \vdots & \vdots \\
\hline c^{\prime} & d^{\prime} \\
\hline a^{\prime} & 1 \\
\hline
\end{array}
$$

where $a^{\prime}=a+1, c^{\prime}=c+1$, and $d^{\prime}=d+1$. If $c<a<d$ or $d<a<c$, then

$$
s_{1}(T)=\begin{array}{c|c|c}
\vdots & \vdots \\
\hline d & c \\
\hline 2 n & a \\
\hline
\end{array}, \quad s_{1}(\operatorname{cyc}(T))=\begin{array}{|c|c|c}
\vdots & \vdots \\
\hline d^{\prime} & c^{\prime} \\
\hline 1 & a^{\prime}
\end{array}, \quad \text { and } \quad \operatorname{cyc}\left(s_{1}(T)\right)=\begin{array}{ccc}
\hline \vdots & \vdots \\
\hline d^{\prime} & c^{\prime} \\
\hline 1 & a^{\prime} \\
\hline
\end{array},
$$

where the portions of the fillings above the bottom 2 rows agree by induction. Otherwise,

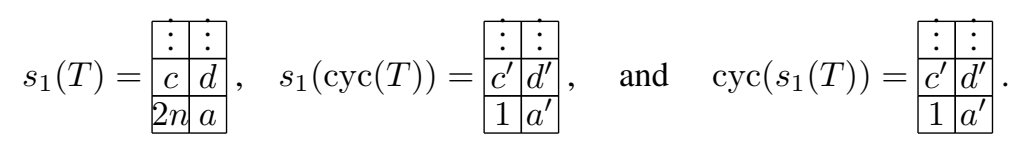

Therefore, $s_{1} \circ \mathrm{cyc}=\operatorname{cyc} \circ s_{1}$.

Proposition 5.6 Let $T \in \mathcal{F}_{\left(2^{n}\right)}$ for some $n \geq 1$ where $2 n$ is in the bottom row of $T$. Let $S=s_{1}(\operatorname{cyc}(T))$. Then $\operatorname{comaj}_{\left(2^{n}\right)}(S)=\operatorname{comaj}_{\left(2^{n}\right)}(T)-(n-1)$ and $\operatorname{inv}_{\left(2^{n}\right)}(S)=\operatorname{inv}_{\left(2^{n}\right)}(T)$.

Proof: First notice that when $2 n$ is in the bottom row of $T$,

$$
\operatorname{inv}_{\left(2^{n}\right)}(\operatorname{cyc}(T))=\operatorname{inv}_{\left(2^{n}\right)}(T)+ \begin{cases}1 & \text { if } 2 n \text { is in column } 2 \\ -1 & \text { if } 2 n \text { is in column } 1 .\end{cases}
$$

Since the entry $2 n$ in $T$ becomes 1 in $\operatorname{cyc}(T)$,

$$
\operatorname{comaj}_{\left(2^{n}\right)}(\operatorname{cyc}(T))=\operatorname{comaj}_{\left(2^{n}\right)}(T)-(n-1)
$$


because the ascent which occurs in $T$ between $2 n$ and the entry above it is lost. It follows from 3.4 that

$$
\operatorname{inv}_{\left(2^{n}\right)}(S)=\operatorname{inv}_{\left(2^{n}\right)}\left(s_{1}(\operatorname{cyc}(T))\right)=\operatorname{inv}_{\left(2^{n}\right)}(T)
$$

and

$$
\operatorname{comaj}_{\left(2^{n}\right)}(S)=\operatorname{comaj}_{\left(2^{n}\right)}\left(s_{1}(\operatorname{cyc}(T))\right)=\operatorname{comaj}_{\left(2^{n}\right)}(T)-(n-1) .
$$

Example 5.7 For $n=4$ and

$$
T=\begin{array}{|l|l|}
\hline 6 & 1 \\
\hline 7 & 5 \\
\hline 4 & 2 \\
\hline 3 & 8 \\
\hline
\end{array}
$$

we have

$$
\operatorname{cyc}(T)=\begin{array}{|l|l|}
\hline 7 & 2 \\
\hline 8 & 6 \\
\hline 5 & 3 \\
\hline 4 & 1 \\
\hline
\end{array}
$$

and

$$
S=s_{1}(\operatorname{cyc}(T))=\begin{array}{|l|l|}
\hline 7 & 2 \\
\hline 8 & 6 \\
\hline 3 & 5 \\
\hline 1 & 4 \\
\hline
\end{array} .
$$

Then $\operatorname{comaj}_{\left(2^{4}\right)}(T)=5$, $\operatorname{comaj}_{\left(2^{4}\right)}(S)=2$, and $\operatorname{inv}_{\left(2^{4}\right)}(T)=\operatorname{inv}_{\left(2^{4}\right)}(S)=2$.

\section{Recursions for Two-Column Shapes}

In this section, we consider what happens when we fix the bottom row of the fillings of $\left(2^{n}\right)$ to be $1 \mid a$. This will lead to a recursion characterizing the polynomials $F_{\left(2^{n}\right)}(q, t)$.

Definition 6.1 For $n \geq 1$ and $2 \leq a \leq 2 n$, set $\mathcal{F}_{\left(2^{n}\right), a}=\left\{T \in \mathcal{F}_{\left(2^{n}\right)}:\right.$ Thas bottom row $\left.1 \mid a\right\}$. Define

$$
R_{\left(2^{n}\right), a}=R_{\left(2^{n}\right), a}(q, t)=\sum_{T \in \mathcal{F}_{\left(2^{n}\right), a}} q^{\operatorname{inv}_{\left(2^{n}\right)}(T)} t^{\operatorname{comaj}_{\left(2^{n}\right)}(T)} .
$$

\subsection{Augmentation}

Definition 6.2 For $n \geq 1, T \in \mathcal{F}_{\left(2^{n}\right)}$ and $2 \leq a \leq 2 n+2$, we define the a-augmentation of $T$ to be the filling $A_{a}(T) \in \mathcal{F}_{\left(2^{n+1}\right), a}$ which is obtained by first relabeling the entries of $T$ as follows: $c \in T$ is replaced by $c+1$ if $c<a-1$ and by $c+2$ if $c \geq a-1$; and then placing the relabeled filling over the new bottom row $1 \mid a$.

Example 6.3 For $a=3$ and $T \in \mathcal{F}_{\left(2^{3}\right)}$ given by

$$
T=\begin{array}{|l|l|}
\hline 3 & 5 \\
\hline 6 & 4 \\
\hline 1 & 2 \\
\hline
\end{array}
$$


we have

$$
A_{3}(T)=\begin{array}{|l|l|}
\hline 5 & 7 \\
\hline 8 & 6 \\
\hline 2 & 4 \\
\hline 1 & 3 \\
\hline
\end{array} .
$$

We consider next what $A_{a}$ does to inv ${ }_{\mu}$ and $\operatorname{comaj}_{\mu}$ and how it interacts with cyc and $s_{1}$. Here and below, for any statement $P$, we write $\chi(P)=1$ if $P$ is true and $\chi(P)=0$ if $P$ is false.

Proposition 6.4 For all $n \geq 2$, all a with $2 \leq a \leq 2 n$, and all $T \in \mathcal{F}_{\left(2^{n-1}\right)}$ with bottom row $x \mid z$,

(a) $\operatorname{comaj}_{\left(2^{n}\right)}\left(A_{a}(T)\right)=\operatorname{comaj}_{\left(2^{n-1}\right)}(T)+(n-1) \chi(z<a-1)$;

(b) $\operatorname{inv}_{\left(2^{n}\right)}\left(A_{a}(T)\right)=\operatorname{inv}_{\left(2^{n-1}\right)}(T)$.

Proof: Let $T \in \mathcal{F}_{\left(2^{n-1}\right)}$ have bottom row $x z$, and let $2 \leq a \leq 2 n$. The bottom two rows of $A_{a}(T)$ look like:

$$
\begin{array}{|c|c|}
\hline x^{\prime} & z^{\prime} \\
\hline 1 & a \\
\hline
\end{array}
$$

where $x^{\prime}$ is $x+1$ if $x<a-1$ or $x+2$ if $x \geq a-1$, and $z^{\prime}$ is $z+1$ if $z<a-1$ or $z+2$ if $z \geq a-1$. Now $\operatorname{inv}_{\left(2^{n}\right)}\left(A_{a}(T)\right)=\operatorname{inv}_{\left(2^{n-1}\right)}(T)$ since the two entries in the bottom row of $A_{a}(T)$ do not form an inversion triple, and $1, x^{\prime}, z^{\prime}$ form an inversion triple in $A_{a}(T)$ iff $\infty, x, z$ form an inversion triple in $T$. The ascents in the top $n-1$ rows of $A_{a}(T)$ are the same as the ascents in $T$. There cannot be an ascent between the two lowest cells in column 1 of $A_{a}(T)$ since the lowest cell in this column contains 1. If $a$ is greater than the entry directly above it in $A_{a}(T)$, that entry is $z+1$, giving a new ascent, so $\operatorname{comaj}_{\left(2^{n}\right)}\left(A_{a}(T)\right)=\operatorname{comaj}_{\left(2^{n-1}\right)}(T)+n-1$. If $a$ is less than the entry directly above it in $A_{a}(T)$, that entry must be $z+2$, and thus comaj ${ }_{\left(2^{n}\right)}\left(A_{a}(T)\right)=\operatorname{comaj}_{\left(2^{n-1}\right)}(T)$.

Definition 6.5 For all $n \geq 2$ and all $a$ with $2 \leq a \leq 2 n$, define $\overline{\mathrm{cyc}}: \mathcal{F}_{\left(2^{n}\right), a} \rightarrow \mathcal{F}_{\left(2^{n}\right), a}$ by $\overline{\mathrm{cyc}}=$ $A_{a} \circ$ cyc $\circ A_{a}^{-1}$. Similarly, define $\bar{s}_{1}: \mathcal{F}_{\left(2^{n}\right), a} \rightarrow \mathcal{F}_{\left(2^{n}\right), a}$ by $\bar{s}_{1}=A_{a} \circ s_{1} \circ A_{a}^{-1}$.

Proposition 6.6 For all $n, a, b, j, T$ with $n \geq 2,2 \leq a \leq 2 n, 2 \leq b \leq 2 n-2,0 \leq j \leq 2 n-2-b$, and $T \in \mathcal{F}_{\left(2^{n-1}\right), b}$ :

(a) $\operatorname{comaj}_{\left(2^{n}\right)}\left(\overline{\operatorname{cyc}}^{j}\left(A_{a}(T)\right)\right)=\operatorname{comaj}_{\left(2^{n-1}\right)}(T)+j+(n-1) \chi(j<a-b-1)$;

(b) $\operatorname{inv}_{\left(2^{n}\right)}\left(\overline{\operatorname{cyc}}^{j}\left(A_{a}(T)\right)\right)=\operatorname{inv}_{\left(2^{n-1}\right)}(T)$;

(c) $\operatorname{comaj}_{\left(2^{n}\right)}\left(\overline{\operatorname{cyc}}^{j}\left(\bar{s}_{1}\left(A_{a}(T)\right)\right)\right)=\operatorname{comaj}_{\left(2^{n-1}\right)}(T)+j+(n-1) \chi(j<a-2)$;

(d) $\operatorname{inv}_{\left(2^{n}\right)}\left(\overline{\operatorname{cyc}}^{j}\left(\bar{s}_{1}\left(A_{a}(T)\right)\right)\right)=\operatorname{inv}_{\left(2^{n-1}\right)}(T)+1$.

Proof: Let $T \in \mathcal{F}_{\left(2^{n-1}\right), b}$. Then, by 5.3 and 6.4

$$
\begin{aligned}
\operatorname{comaj}_{\left(2^{n}\right)}\left(\overline{\operatorname{cyc}}^{j}\left(A_{a}(T)\right)\right) & =\operatorname{comaj}_{\left(2^{n}\right)}\left(A_{a}\left(\operatorname{cyc}^{j}(T)\right)\right. \\
& =\operatorname{comaj}_{\left(2^{n-1}\right)}\left(\operatorname{cyc}^{j}(T)\right)+(n-1) \chi(b+j<a-1) \\
& =\operatorname{comaj}_{\left(2^{n-1}\right)}(T)+j+(n-1) \chi(j<a-b-1) .
\end{aligned}
$$


By 5.3 and $6.4 \operatorname{inv}_{\left(2^{n}\right)}\left(\overline{\operatorname{cyc}}^{j}\left(A_{a}(T)\right)\right)=\operatorname{inv}_{\left(2^{n-1}\right)}(T)$.

Similarly, using $5.5,6.4$ and 5.3 , we find that

$$
\begin{aligned}
\operatorname{comaj}_{\left(2^{n}\right)}\left(\overline{\operatorname{cyc}}^{j}\left(\bar{s}_{1}\left(A_{a}(T)\right)\right)\right) & =\operatorname{comaj}_{\left(2^{n}\right)}\left(A_{a}\left(\operatorname{cyc}^{j}\left(s_{1}(T)\right)\right)\right) \\
& =\operatorname{comaj}_{\left(2^{n}\right)}\left(A_{a}\left(s_{1}\left(\operatorname{cyc}^{j}(T)\right)\right)\right) \\
& =\operatorname{comaj}_{\left(2^{n-1}\right)}\left(\operatorname{cyc}^{j}(T)\right)+(n-1) \chi(1+j<a-1) \\
& =\operatorname{comaj}_{\left(2^{n-1}\right)}(T)+j+(n-1) \chi(j<a-2) .
\end{aligned}
$$

By 6.4 5.3 and 3.4. $\operatorname{inv}_{\left(2^{n}\right)}\left(\overline{\operatorname{cyc}}^{j}\left(\bar{s}_{1}\left(A_{a}(T)\right)\right)\right)=\operatorname{inv}_{\left(2^{n-1}\right)}(T)+1$.

Proposition 6.7 For all $n \geq 1$ and $2 \leq a \leq 2 n, R_{\left(2^{n}\right), 2 n-a+2}=t^{n-a+1} R_{\left(2^{n}\right), a}$.

Proof: It suffices to prove the result for $2 \leq a \leq n$. Note that $s_{1} \circ \operatorname{cyc}^{2 n-a+1}$ is a bijection from $\mathcal{F}_{\left(2^{n}\right), a}$ onto $\mathcal{F}_{\left(2^{n}\right), 2 n-a+2}$. For $T \in \mathcal{F}_{\left(2^{n}\right), a}, 5.3$ and 5.6 give

$$
\begin{gathered}
\operatorname{comaj}_{\left(2^{n}\right)}\left(s_{1}\left(\operatorname{cyc}^{2 n-a+1}(T)\right)\right)=\operatorname{comaj}_{\left(2^{n}\right)}(T)+2 n-a-(n-1)=\operatorname{comaj}_{\left(2^{n}\right)}(T)+n-a+1 \\
\operatorname{inv}_{\left(2^{n}\right)}\left(s_{1}\left(\operatorname{cyc}^{2 n-a+1}(T)\right)\right)=\operatorname{inv}_{\left(2^{n}\right)}(T) .
\end{gathered}
$$

\subsection{Recursions for Two-Column Rectangles}

We can compute $R_{\left(2^{n}\right), a}$ for $2 \leq a \leq n+1$ by the recursion below. A nicer version of this recursion appears in 6.10 below.

Proposition 6.8 For all $n \geq 2$ and $2 \leq a \leq n+1$,

$$
\begin{aligned}
R_{\left(2^{n}\right), a}=[n-1]_{t} & \left(\sum_{b=2}^{n-1}\left(t^{(a-b-1)^{+}}+t^{n-b}+q t^{a-2}+q t^{n-1-(b+1-a)^{+}}\right) R_{\left(2^{n-1}\right), b}\right. \\
& \left.+\left(1+q t^{a-2}\right) R_{\left(2^{n-1}\right), n}\right)
\end{aligned}
$$

where $R_{(2), 2}=1$ and $x^{+}=\max \{x, 0\}$.

Proof: To prove the theorem bijectively, we will decompose $\mathcal{F}_{\left(2^{n}\right), a}$ into a collection of disjoint sets $X_{2}, \ldots, X_{n}, Y_{2}, \ldots, Y_{n}$, such that the generating function for $X_{b}$ is $[n-1]_{t}\left(t^{(a-b-1)^{+}}+t^{n-b}\right) R_{\left(2^{n-1}\right), b}$ when $2 \leq b<n$, and $[n-1]_{t} R_{\left(2^{n-1}\right), n}$ when $b=n$. Similarly, the generating function for $Y_{b}$ is $[n-1]_{t}\left(q t^{a-2}+q t^{n-1-(b+1-a)^{+}}\right) R_{\left(2^{n-1}\right), b}$ when $2 \leq b<n$, and $[n-1]_{t} q t^{a-2} R_{\left(2^{n-1}\right), n}$ when $b=n$.

For $2 \leq b \leq n$, define

$$
\begin{aligned}
X_{b}=\left\{T \in \mathcal{F}_{\left(2^{n}\right), a}\right. & : \text { for some } U \in \mathcal{F}_{\left(2^{n-1}\right), b}, T=\overline{\operatorname{cyc}}^{i}\left(A_{a}(U)\right) \text { where } 0 \leq i \leq 2 n-2-b \\
& \text { or } \left.T=\overline{\operatorname{cyc}}^{j}\left(\bar{s}_{1}\left(A_{a}(U)\right)\right) \text { for } 2 n-1-b \leq j \leq 2 n-3\right\}
\end{aligned}
$$


and

$$
\begin{gathered}
Y_{b}=\left\{T \in \mathcal{F}_{\left(2^{n}\right), a}: \text { for some } U \in \mathcal{F}_{\left(2^{n-1}\right), b}, T=\overline{\operatorname{cyc}}^{i}\left(\bar{s}_{1}\left(A_{a}(U)\right)\right) \text { where } 0 \leq i \leq 2 n-2-b\right. \\
\text { or } \left.T=\overline{\operatorname{cyc}}^{j}\left(A_{a}(U)\right) \text { for } 2 n-1-b \leq j \leq 2 n-3\right\} .
\end{gathered}
$$

Using 3.2, 5.2, and 5.5, one sees that each $T \in \mathcal{F}_{\left(2^{n}\right), a}$ can be written as $\overline{\operatorname{cyc}}^{j}\left(\bar{s}_{1}^{\epsilon}\left(A_{a}(U)\right)\right)$ for exactly one choice of $j \in\{0,1, \ldots, 2 n-3\}, \epsilon \in\{0,1\}, b \in\{2, \ldots, n\}$, and $U \in \mathcal{F}_{\left(2^{n-1}\right), b}$. To find $j, \epsilon, b$, and $U$, delete the bottom row of $T$, renumber the remaining entries to be $\{1,2, \ldots, 2 n-2\}$, cyclically shift down until 1 appears in the new bottom row, and then apply $s_{1}$ if needed to get 1 in the lower-left corner. For example, if $n=5$ and $a=4$, the filling

$$
T=\begin{array}{|c|c|}
\hline 9 & 3 \\
\hline 6 & 10 \\
\hline 2 & 7 \\
\hline 5 & 8 \\
\hline 1 & 4 \\
\hline
\end{array}
$$

can be written as $\overline{\operatorname{cyc}}^{2}\left(A_{4}(U)\right)$ where

$$
U=\begin{array}{|l|l|}
\hline 5 & 8 \\
\hline 2 & 6 \\
\hline 7 & 3 \\
\hline 1 & 4 \\
\hline
\end{array}
$$

It follows that $\mathcal{F}_{\left(2^{n}\right), a}$ is the disjoint union of the sets $X_{b}$ and $Y_{b}$ just defined.

By 5.6 and 6.6. $\operatorname{inv}_{\left(2^{n}\right)}\left(\overline{\operatorname{cyc}}^{i}\left(A_{a}(U)\right)\right)=\operatorname{inv}_{\left(2^{n}\right)}\left(\overline{\operatorname{cyc}}^{j}\left(\bar{s}_{1}\left(A_{a}(U)\right)\right)\right)=\operatorname{inv}_{\left(2^{n-1}\right)}(U)$ for all $0 \leq i \leq$ $2 n-2-b$ and all $2 n-1-b \leq j \leq 2 n-3$. Similarly,

$$
\operatorname{inv}_{\left(2^{n}\right)}\left(\overline{\operatorname{cyc}}^{i}\left(\bar{s}_{1}\left(A_{a}(U)\right)\right)\right)=\operatorname{inv}_{\left(2^{n}\right)}\left(\overline{\operatorname{cyc}}^{j}\left(A_{a}(U)\right)\right)=\operatorname{inv}_{\left(2^{n-1}\right)}(U)+1
$$

for all $i, j$ in the indicated ranges. Before continuing the proof, we give an example that illustrates the main ideas in the calculations that follow.

Example 6.9 For $n=4$, $a=4$, and $b=3$, consider

$$
U=\begin{array}{|l|l|}
\hline 2 & 6 \\
\hline 5 & 4 \\
\hline 1 & 3 \\
\hline
\end{array} \quad \text { and } A_{4}(U)=\begin{array}{|l|l|}
\hline 3 & 8 \\
\hline 7 & 6 \\
\hline 2 & 5 \\
\hline 1 & 4 \\
\hline
\end{array} .
$$

Notice that $q^{\operatorname{inv}_{\left(2^{3}\right)}(U)} t^{\operatorname{comaj}_{\left(2^{3}\right)}(U)}=q^{2} t^{1}$. In Figure 2, the fillings in bold are elements of $X_{3}$ derived from $U$, while the rest are elements of $Y_{3}$ derived from $U$. The generating functions for these elements in $X_{3}$ and $Y_{3}$ are $[3]_{t}(1+t) q^{2} t^{1}$ and $[3]_{t}\left(q t^{2}+q t^{3}\right) q^{2} t^{1}$, respectively.

Returning to the proof, we first consider the case where $2 \leq b<n$ and $a \leq b+1$. Let $U \in \mathcal{F}_{\left(2^{n-1}\right), b}$. By 6.6. $\operatorname{comaj}_{\left(2^{n}\right)}\left(\overline{\mathrm{cyc}}^{i}\left(A_{a}(U)\right)\right)=\operatorname{comaj}_{\left(2^{n-1}\right)}(U)+i$ for $0 \leq i \leq 2 n-2-b$. For $2 n-1-b \leq i \leq 2 n-3$, $\operatorname{comaj}_{\left(2^{n}\right)}\left(\overline{\operatorname{cyc}}^{i}\left(\bar{s}_{1}\left(A_{a}(U)\right)\right)\right)=\operatorname{comaj}_{\left(2^{n-1}\right)}(U)+i-(n-1)$ by 6.6 and 5.6 since $2 n-b>a$. 


\begin{tabular}{|c|c|c|c|c|c|c|c|c|c|c|}
\hline \begin{tabular}{|l|l|}
3 & 8 \\
\end{tabular} & & \begin{tabular}{|l|l|}
5 & 2 \\
\end{tabular} & & \begin{tabular}{|l|l|}
6 & 3 \\
\end{tabular} & & \begin{tabular}{|l|l}
7 & 5 \\
\end{tabular} & & \begin{tabular}{|l|l|}
8 & 6 \\
\end{tabular} & & \begin{tabular}{|l|l}
2 & 7
\end{tabular} \\
\hline \begin{tabular}{|l|l|}
7 & 6 \\
\end{tabular} & $\overline{\mathrm{cyc}}$ & \begin{tabular}{|l|l|}
8 & 7 \\
\end{tabular} & $\overline{\mathrm{cyc}}$ & \begin{tabular}{|l|l|}
2 & 8 \\
\end{tabular} & $\overline{\mathrm{cyc}}$ & \begin{tabular}{|l|l}
3 & 2 \\
\end{tabular} & $\overline{\mathrm{cyc}}$ & \begin{tabular}{|l|l|}
5 & 3 \\
\end{tabular} & $\overline{\mathrm{cyc}}$ & \begin{tabular}{|l|l}
6 & 5 \\
\end{tabular} \\
\hline & & \begin{tabular}{|l|l|}
3 & 6 \\
\end{tabular} & & \begin{tabular}{|l|l|}
5 & 7 \\
\end{tabular} & & \begin{tabular}{|l|l|}
6 & 8 \\
\end{tabular} & & \begin{tabular}{|l|l|}
7 & 2 \\
\end{tabular} & & \begin{tabular}{|l|l|}
8 & 3 \\
\end{tabular} \\
\hline \begin{tabular}{|l|l|}
1 & 4 \\
\end{tabular} & & \begin{tabular}{|l|l|}
1 & 4 \\
\end{tabular} & & \begin{tabular}{|l|l|}
1 & 4 \\
\end{tabular} & & \begin{tabular}{|l|l|}
1 & 4 \\
\end{tabular} & & \begin{tabular}{|l|l|}
1 & 4 \\
\end{tabular} & & \begin{tabular}{|l|l|}
1 & 4 \\
\end{tabular} \\
\hline$q^{2} t$ & & $q^{2} t^{2}$ & & $q^{2} t^{3}$ & & $q^{2} t^{4}$ & & $q^{3} t^{5}$ & & $q^{3} t^{6}$ \\
\hline$\downarrow \bar{s}_{1}$ & & $\uparrow \bar{s}_{1}$ & & $\uparrow \bar{s}_{1}$ & & $\uparrow \bar{s}_{1}$ & & $\uparrow \bar{s}_{1}$ & & $\uparrow \bar{s}_{1}$ \\
\hline \begin{tabular}{|l|l|}
3 & 8 \\
\end{tabular} & & \begin{tabular}{|l|l|}
5 & 2 \\
\end{tabular} & & \begin{tabular}{|l|l|l|}
6 & 3 \\
\end{tabular} & & \begin{tabular}{|l|l}
7 & 5 \\
\end{tabular} & & \begin{tabular}{|l|l|}
8 & 6 \\
\end{tabular} & & \begin{tabular}{|l|l}
2 & 7 \\
\end{tabular} \\
\hline \begin{tabular}{|l|l|}
7 & 6 \\
\end{tabular} & $\overline{\mathrm{cyc}}$ & \begin{tabular}{|l|l|}
8 & 7 \\
\end{tabular} & $\overline{\mathrm{cyc}}$ & \begin{tabular}{|l|l|}
2 & 8 \\
\end{tabular} & $\overline{\mathrm{cyc}}$ & \begin{tabular}{|l|l}
3 & 2 \\
\end{tabular} & $\overline{\mathrm{cyc}}$ & \begin{tabular}{|l|l|}
5 & 3 \\
\end{tabular} & $\overline{\mathrm{cyc}}$ & \begin{tabular}{|l|l}
6 & 5 \\
\end{tabular} \\
\hline \begin{tabular}{|l|l|}
5 & 2 \\
\end{tabular} & & \begin{tabular}{|l|l|}
6 & 3 \\
\end{tabular} & & \begin{tabular}{|l|l|} 
& 5 \\
\end{tabular} & & $\begin{array}{ll}8 & 6 \\
\end{array}$ & & \begin{tabular}{|l|l}
2 & 7 \\
\end{tabular} & & \begin{tabular}{l|l}
3 & 8 \\
\end{tabular} \\
\hline \begin{tabular}{|l|l|}
1 & 4 \\
\end{tabular} & & \begin{tabular}{|l|l|}
1 & 4 \\
\end{tabular} & & \begin{tabular}{|l|l|l|}
1 & 4 \\
\end{tabular} & & \begin{tabular}{|l|l|}
1 & 4 \\
\end{tabular} & & \begin{tabular}{|l|l|}
1 & 4 \\
\end{tabular} & & \begin{tabular}{|l|l}
1 & 4 \\
\end{tabular} \\
\hline$q^{3} t^{4}$ & & $q^{3} t^{5}$ & & $q^{3} t^{3}$ & & $q^{3} t^{4}$ & & $q^{2} t^{2}$ & & $q^{2} t^{3}$ \\
\hline
\end{tabular}

Fig. 2: The elements of $X_{3}$ and $Y_{3}$ generated by $U$.

We now compute $\sum_{i=0}^{2 n-3} t^{\operatorname{comaj}_{\left(2^{n}\right)}\left(\overline{\mathrm{cyc}}^{i}\left(A_{a}(U)\right)\right)}$ by splitting the sum into the ranges $0 \leq i \leq n-2$, $n-1 \leq i \leq 2 n-2-b$, and $2 n-1-b \leq i \leq 2 n-3$. For $i$ in the first range, we compute

$$
\sum_{i=0}^{n-2} t^{\operatorname{comaj}_{\left(2^{n}\right)}\left(\overline{\mathrm{cyc}}^{i}\left(A_{a}(U)\right)\right)}=[n-1]_{t} t^{\operatorname{comaj}_{\left(2^{n-1}\right)}(U)}=[n-1]_{t} t^{(a-b-1)^{+}} t^{\operatorname{comaj}_{\left(2^{n-1}\right)}(U)} .
$$

For the remaining values of $i$, we obtain

$$
\begin{aligned}
& \sum_{i=n-1}^{2 n-2-b} t^{\operatorname{comaj}_{\left(2^{n}\right)}\left(\overline{\operatorname{cyc}}^{i}\left(A_{a}(U)\right)\right)}+\sum_{i=2 n-1-b}^{2 n-3} t^{\operatorname{comaj}_{\left(2^{n}\right)}\left(\overline{\operatorname{cyc}}^{i}\left(\bar{s}_{1}\left(A_{a}(U)\right)\right)\right)} \\
&=t^{\operatorname{comaj}_{\left(2^{n-1}\right)}(U)}\left(\sum_{i=n-1}^{2 n-2-b} t^{i}+\sum_{i=2 n-1-b}^{2 n-3} t^{i-(n-1)}\right)=t^{\operatorname{comaj}_{\left(2^{n-1}\right)}(U)}\left(\sum_{i=n-1}^{2 n-2-b} t^{i}+\sum_{i=n-b}^{n-2} t^{i}\right) \\
&=[n-1]_{t} t^{n-b+\operatorname{comaj}_{\left(2^{n-1}\right)}(U)}
\end{aligned}
$$

Thus, the generating function for $X_{b}$ is $[n-1]_{t}\left(t^{(a-b-1)^{+}}+t^{n-b}\right) R_{\left(2^{n-1}\right), b}$. Similarly, we can use 6.6 and 5.6 to obtain:

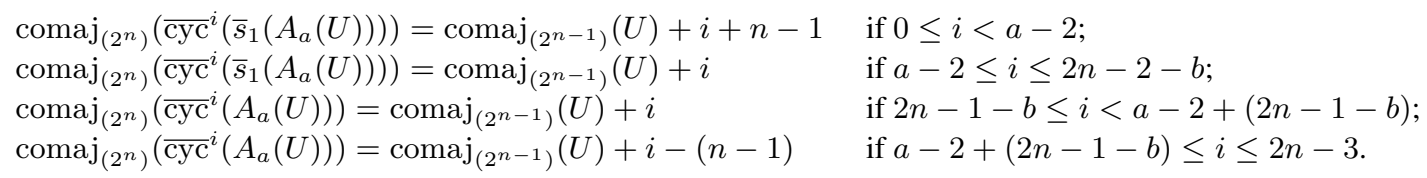


Then

$$
\begin{aligned}
& \sum_{i=0}^{2 n-3} t^{\operatorname{comaj}_{\left(2^{n}\right)}\left(\overline{c y c}^{i}\left(\bar{s}_{1}\left(A_{a}(U)\right)\right)\right)}=\sum_{i=0}^{a-3} t^{\operatorname{comaj}_{\left(2^{n-1}\right)}(U)+i+n-1}+\sum_{i=a-2}^{n-2} t^{\operatorname{comaj}_{\left(2^{n-1}\right)}(U)+i} \\
& \quad+\sum_{i=n-1}^{2 n-2-b} t^{\operatorname{comaj}_{\left(2^{n-1}\right)}(U)+i}+\sum_{i=2 n-b-1}^{a+2 n-b-4} t^{\operatorname{comaj}_{\left(2^{n-1}\right)}(U)+i}+\sum_{i=a+2 n-b-3}^{2 n-3} t^{\operatorname{comaj}_{\left(2^{n-1}\right)}(U)+i-(n-1)} \\
& \quad=\left(\sum_{i=a-2}^{a+n-4} t^{i}+\sum_{i=n-1}^{a+2 n-b-4} t^{i}+\sum_{i=a+n-b-2}^{n-2} t^{i} t^{\operatorname{comaj}_{\left(2^{n-1}\right)}(U)}\right. \\
& =[n-1]_{t}\left(t^{a-2}+t^{n-1-(b+1-a)}\right) t^{\operatorname{comaj}_{\left(2^{n-1}\right)}(U)}
\end{aligned}
$$

So the generating function for $Y_{b}$ in this case is $[n-1]_{t} q\left(t^{a-2}+t^{n-1-(b+1-a)^{+}}\right) R_{\left(2^{n-1}\right), b}$.

Next we look at what happens when $b+1<a, 2 \leq b<n$, and $2 \leq a \leq n+1$. Let $U \in \mathcal{F}_{\left(2^{n-1}\right), b}$. Then, by 6.6 and 5.6 .

$$
\begin{array}{ll}
\operatorname{comaj}_{\left(2^{n}\right)}\left(\overline{\operatorname{cyc}}^{i}\left(A_{a}(U)\right)\right)=\operatorname{comaj}_{\left(2^{n-1}\right)}(U)+i+n-1 & \text { if } 0 \leq i<a-b-1 \\
\operatorname{comaj}_{\left(2^{n}\right)}\left(\overline{\operatorname{cyc}}^{i}\left(A_{a}(U)\right)\right)=\operatorname{comaj}_{\left(2^{n-1}\right)}(U)+i & \text { if } a-b-1 \leq i \leq 2 n-b-2 \\
\operatorname{comaj}_{\left(2^{n}\right)}\left(\overline{\operatorname{cyc}}^{i}\left(\bar{s}_{1}\left(A_{a}(U)\right)\right)\right)=\operatorname{comaj}_{\left(2^{n-1}\right)}(U)+i-(n-1) & \text { if } 2 n-b-1 \leq i \leq 2 n-3
\end{array}
$$

Then

$$
\begin{aligned}
\sum_{i=0}^{2 n-3} t^{\operatorname{comaj}_{\left(2^{n}\right)}\left(\overline{c y c}^{i}\left(A_{a}(U)\right)\right)}= & \sum_{i=0}^{a-b-2} t^{\operatorname{comaj}_{\left(2^{n-1}\right)}(U)+i+n-1}+\sum_{i=a-b-1}^{n-2} t^{\operatorname{comaj}_{\left(2^{n-1}\right)}(U)+i} \\
& +\sum_{i=n-1}^{2 n-b-2} t^{\operatorname{comaj}_{\left(2^{n-1}\right)}(U)+i}+\sum_{i=2 n-b-1}^{2 n-3} t^{\operatorname{comaj}_{\left(2^{n-1}\right)}(U)+i-(n-1)} \\
& =\left(\sum_{i=n-1}^{n+a-b-3} t^{i}+\sum_{i=a-b-1}^{n-2} t^{i}+\sum_{i=n-1}^{2 n-b-2} t^{i}+\sum_{i=n-b}^{n-2} t^{i}\right) t^{\operatorname{comaj}_{\left(2^{n-1}\right)}(U)} \\
& =[n-1]_{t}\left(t^{(a-b-1)}+t^{n-b}\right) t^{\mathrm{comaj}_{\left(2^{n-1}\right)}(U)} .
\end{aligned}
$$

Thus, the generating function for $X_{b}$ in this case is $[n-1]_{t}\left(t^{(a-b-1)^{+}}+t^{n-b}\right) R_{\left(2^{n-1}\right), b}$.

Similarly, for $Y_{b}$, we have

$$
\begin{array}{ll}
\operatorname{comaj}_{\left(2^{n}\right)}\left(\overline{\operatorname{cyc}}^{i}\left(\bar{s}_{1}\left(A_{a}(U)\right)\right)\right)=\operatorname{comaj}_{\left(2^{n-1}\right)}(U)+i+n-1 & \text { if } 0 \leq i<a-2 \\
\operatorname{comaj}_{\left(2^{n}\right)}\left(\overline{\operatorname{cyc}}^{i}\left(\bar{s}_{1}\left(A_{a}(U)\right)\right)\right)=\operatorname{comaj}_{\left(2^{n-1}\right)}(U)+i & \text { if } a-2 \leq i \leq 2 n-2-b \\
\operatorname{comaj}_{\left(2^{n}\right)}\left(\overline{\operatorname{cyc}}^{i}\left(A_{a}(U)\right)\right)=\operatorname{comaj}_{\left(2^{n-1}\right)}(U)+i & \text { if } 2 n-1-b \leq i \leq 2 n-3
\end{array}
$$


Then

$$
\begin{aligned}
\sum_{i=0}^{2 n-3} t^{\operatorname{comaj}_{\left(2^{n}\right)}\left(\overline{\operatorname{cyc}}^{i}\left(\bar{s}_{1}\left(A_{a}(U)\right)\right)\right)=} & \sum_{i=0}^{a-3} t^{\operatorname{comaj}_{\left(2^{n-1}\right)}(U)+i+n-1}+\sum_{i=a-2}^{n-2} t^{\operatorname{comaj}_{\left(2^{n-1}\right)}(U)+i} \\
& \quad+\sum_{i=n-1}^{2 n-2-b} t^{\operatorname{comaj}_{\left(2^{n-1}\right)}(U)+i}+\sum_{i=2 n-1-b}^{2 n-3} t^{\operatorname{comaj}_{\left(2^{n-1}\right)}(U)+i} \\
= & {[n-1]_{t}\left(t^{a-2}+t^{n-1}\right) t^{\operatorname{comaj}_{\left(2^{n-1}\right)}(U)} }
\end{aligned}
$$

So the generating function for $Y_{b}$ in this case is $[n-1]_{t} q\left(t^{a-2}+t^{n-1}\right) R_{\left(2^{n-1}\right), b}$. Combining all of the above gives us that, for $2 \leq b<n$, the generating function for $X_{b}$ is $[n-1]_{t}\left(t^{(a-b-1)^{+}}+t^{n-b}\right) R_{\left(2^{n-1}\right), b}$ and the generating function for $Y_{b}$ is $[n-1]_{t} q\left(t^{a-2}+t^{n-1-(b+1-a)^{+}}\right) R_{\left(2^{n-1}\right), b}$.

When $b=n$ and $U \in \mathcal{F}_{\left(2^{n-1}\right), n}$,

$$
A_{a}(U), \overline{\operatorname{cyc}}\left(A_{a}(U)\right), \ldots, \overline{\operatorname{cyc}}^{n-2}\left(A_{a}(U)\right) \in X_{n}
$$

and

$$
\bar{s}_{1}\left(A_{a}(U)\right), \overline{\operatorname{cyc}}\left(\bar{s}_{1}\left(A_{a}(U)\right)\right), \ldots, \overline{\operatorname{cyc}}^{n-2}\left(\bar{s}_{1}\left(A_{a}(U)\right)\right) \in Y_{n} .
$$

Since $a-n-1 \leq 0$,

$$
\operatorname{comaj}_{\left(2^{n}\right)}\left(\overline{\operatorname{cyc}}^{i}\left(A_{a}(U)\right)\right)=\operatorname{comaj}_{\left(2^{n-1}\right)}(U)+i,
$$

so the generating function for $X_{n}$ is $[n-1]_{t} R_{\left(2^{n-1}\right), n}$. To obtain the generating function for $Y_{n}$, we note that

$$
\begin{array}{ll}
\operatorname{comaj}_{\left(2^{n}\right)}\left(\overline{\operatorname{cyc}}^{i}\left(\bar{s}_{1}\left(A_{a}(U)\right)\right)\right)=\operatorname{comaj}_{\left(2^{n-1}\right)}(U)+i+n-1 & \text { if } 0 \leq i<a-2 \\
\operatorname{comaj}_{\left(2^{n}\right)}\left(\overline{\operatorname{cyc}}^{i}\left(\bar{s}_{1}\left(A_{a}(U)\right)\right)\right)=\operatorname{comaj}_{\left(2^{n-1}\right)}(U)+i & \text { if } a-2 \leq i \leq n-2
\end{array}
$$

Then, as above, the generating function for $Y_{n}$ is $[n-1]_{t}\left(q t^{a-2}\right) R_{\left(2^{n-1}\right), n}$.

We can use 6.7 to obtain the following more streamlined version of the recursion for two-column rectangles.

Theorem 6.10 For all $n \geq 2$ and $2 \leq a \leq 2 n$,

$$
R_{\left(2^{n}\right), a}=[n-1]_{t} \sum_{b=2}^{2 n-2}\left(t^{(a-b-1)^{+}}+q t^{n-1-(b+1-a)^{+}}\right) R_{\left(2^{n-1}\right), b}
$$

where $R_{(2), 2}=1$ and $x^{+}=\max (x, 0)$. 
Proof: First, we use 6.7 to rewrite the recursion from 6.8 for $2 \leq a \leq n+1$. We see that $R_{\left(2^{n}\right), a}$ equals

$$
\begin{aligned}
& {[n-1]_{t}\left(\sum_{b=2}^{n-1}\left(t^{(a-b-1)^{+}}+t^{n-b}+q t^{a-2}+q t^{n-1-(b+1-a)^{+}}\right) R_{\left(2^{n-1}\right), b}+\left(1+q t^{a-2}\right) R_{\left(2^{n-1}\right), n}\right) } \\
&=[n-1]_{t}\left(\sum_{b=2}^{n-1}\left(t^{(a-b-1)^{+}}+q t^{n-1-(b+1-a)^{+}}\right) R_{\left(2^{n-1}\right), b}+\sum_{b=2}^{n-1} t^{b-n}\left(t^{n-b}+q t^{a-2}\right) R_{\left(2^{n-1}\right), 2 n-b}\right. \\
&\left.\quad+\left(1+q t^{a-2}\right) R_{\left(2^{n-1}\right), n}\right) \\
&=[n-1]_{t}\left(\sum_{b=2}^{n}\left(t^{(a-b-1)^{+}}+q t^{n-1-(b+1-a)^{+}}\right) R_{\left(2^{n-1}\right), b}+\sum_{b=2}^{n-1}\left(1+q t^{a+b-n-2}\right) R_{\left(2^{n-1}\right), 2 n-b}\right) \\
&=[n-1]_{t}\left(\sum_{b=2}^{n}\left(t^{(a-b-1)^{+}}+q t^{n-1-(b+1-a)^{+}}\right) R_{\left(2^{n-1}\right), b}+\sum_{b=n+1}^{2 n-2}\left(1+q t^{n-1-(b+1-a)}\right) R_{\left(2^{n-1}\right), b}\right) \\
&=[n-1]_{t} \sum_{b=2}^{2 n-2}\left(t^{(a-b-1)^{+}}+q t^{n-1-(b+1-a)^{+}}\right) R_{\left(2^{n-1}\right), b} .
\end{aligned}
$$

To see that this same recursion works for $n+2 \leq a \leq 2 n$, apply 6.7 again. For $a$ with $n+2 \leq a \leq 2 n$, note that $2 \leq 2 n-a+2 \leq n+1$. Then

$$
\begin{aligned}
R_{\left(2^{n}\right), a} & =t^{a-n-1} R_{\left(2^{n}\right), 2 n-a+2} \\
& =t^{a-n-1}[n-1]_{t} \sum_{b=2}^{2 n-2}\left(t^{(2 n-a+2-b-1)^{+}}+q t^{n-1-(b+1-(2 n-a+2))^{+}}\right) R_{\left(2^{n-1}\right), b} \\
& =[n-1]_{t} \sum_{b=2}^{2 n-2}\left(t^{a-n-1+(2 n-a+2-b-1)^{+}}+q t^{a-2-(b+a-2 n-1)^{+}}\right) R_{\left(2^{n-1}\right), b} .
\end{aligned}
$$

One final application of 6.7, this time to $R_{\left(2^{n-1}\right), b}$ for $2 \leq b \leq 2 n-2$, leads to

$$
\begin{aligned}
R_{\left(2^{n}\right), a} & =[n-1]_{t} t^{n-b} \sum_{b=2}^{2 n-2}\left(t^{a-n-1+(2 n-a+1-(2 n-b))^{+}}+q t^{a-2-(2 n-b+a-2 n-1)^{+}}\right) R_{\left(2^{n-1}\right), b} \\
& =[n-1]_{t} \sum_{b=2}^{2 n-2}\left(t^{a-b-1+(b+1-a)^{+}}+q t^{n-b+a-2-(a-b-1)^{+}}\right) R_{\left(2^{n-1}\right), b} \\
& =[n-1]_{t} \sum_{b=2}^{2 n-2}\left(t^{(a-b-1)^{+}}+q t^{n-1-(b+1-a)^{+}}\right) R_{\left(2^{n-1}\right), b} .
\end{aligned}
$$

Proposition 6.11 For $n \geq 1, F_{\left(2^{n}\right)}(q, t)=R_{\left(2^{n+1}\right), 2}(q, t)$. 
Proof: Note $A_{2}$ is a bijection from $\mathcal{F}_{\left(2^{n}\right)}$ onto $\mathcal{F}_{\left(2^{n+1}\right), 2}$. By 6.4, for $U \in \mathcal{F}_{\left(2^{n}\right)}$,

$$
\operatorname{comaj}_{\left(2^{n+1}\right)}\left(A_{2}(U)\right)=\operatorname{comaj}_{\left(2^{n}\right)}(U) \text { and } \operatorname{inv}_{\left(2^{n+1}\right)}\left(A_{2}(U)\right)=\operatorname{inv}_{\left(2^{n}\right)}(U) .
$$

Using the recursion 6.10 and induction on $n$, we see that $R_{\left(2^{n}\right), a}(q, t)$ is divisible by $[n-1] !_{t}$ for $2 \leq a \leq 2 n$. Then 6.11 shows that $F_{\left(2^{n}\right)}(q, t)$ is indeed divisible by $[n] !_{t}$. This gives the promised combinatorial proof of Theorem 1.1 (b) in the case of two-column rectangles.

\subsection{Recursion for Two Unequal Columns}

Next we discuss a recursion valid for shapes $\mu$ with two unequal columns.

Definition 6.12 For $m \geq 1$ and $n \geq 1$, let $\mu \in \operatorname{Par}(2 n+m)$ with $\mu=\left(1^{m} 2^{n}\right)$. Let $\mathcal{F}_{\mu, a}=\left\{T \in \mathcal{F}_{\mu}\right.$ : T has bottom row $1|a|$ \}, and define

$$
R_{\left(1^{m} 2^{n}\right), a}(q, t)=R_{\left(1^{m} 2^{n}\right), a}=\sum_{T \in \mathcal{F}_{\left(1^{m} 2^{n}\right), a}} q^{\operatorname{inv}_{\left(1^{m} 2^{n}\right)}(T)} t^{\operatorname{comaj}_{\left(1^{m} 2^{n}\right)}(T)} .
$$

To analyze $R_{\left(1^{m} 2^{n}\right), a}$, we need a definition and proposition analogous to 6.2 and 6.4 for two equal columns.

Definition 6.13 For $m \geq 1, n \geq 0, T \in \mathcal{F}_{\left(1^{m} 2^{n}\right)}$ and $2 \leq a \leq m+2 n+2$, we define the a-augmentation of $T$ to be the filling $A_{a}(T) \in \mathcal{F}_{\left(1^{m} 2^{n+1}\right), a}$ which is obtained by first relabeling the entries of $T$ as follows: $c \in T$ is replaced by $c+1$ if $c<a-1$ and by $c+2$ if $c \geq a-1$; and then placing the relabeled filling over the new bottom row $1 \mid \begin{aligned} & 1 \\ & \text {. }\end{aligned}$

Proposition 6.14 For all $m \geq 1, n \geq 1$, all a with $2 \leq a \leq m+2 n$, and all $T \in \mathcal{F}_{\left(1^{m} 2^{n-1}\right)}$ with bottom row $x \mid z$,

(a) $\operatorname{comaj}_{\left(1^{m} 2^{n}\right)}\left(A_{a}(T)\right)=\operatorname{comaj}_{\left(1^{m} 2^{n-1}\right)}(T)+(n-1) \chi(z<a-1)$;

(b) $\operatorname{inv}_{\left(1^{m} 2^{n}\right)}\left(A_{a}(T)\right)=\operatorname{inv}_{\left(1^{m} 2^{n-1}\right)}(T)$.

Since the columns are not of equal height, the inversion flip is not available to sort. Instead, we use the cyclic shift and careful accounting to obtain an increasing bottom row.

Proposition 6.15 Let $m \geq 1, n \geq 0$ and $T \in \mathcal{F}_{\left(1^{m} 2^{n}\right)}$, where $T$ has bottom row $x \mid z$ for some $x>z$. Then cyc $^{m+2 n-x+1}(T)$ will have bottom row $1 \quad b$ where $b=m+2 n-x+1+z$,

$$
\operatorname{comaj}_{\left(1^{m} 2^{n}\right)}\left(\operatorname{cyc}^{m+2 n-x+1}(T)\right)=\operatorname{comaj}_{\left(1^{m} 2^{n}\right)}(T)+n-x+1
$$

and

$$
\operatorname{inv}_{\left(1^{m} 2^{n}\right)}\left(\operatorname{cyc}^{m+2 n-x+1}(T)\right)=\operatorname{inv}_{\left(1^{m} 2^{n}\right)}(T)-1 .
$$

Proof: Let $T \in \mathcal{F}_{\left(1^{m} 2^{n}\right)}$ have bottom row $x \mid z$ with $x>z$. By 5.3 .

$$
\operatorname{comaj}_{\left(1^{m} 2^{n}\right)}\left(\operatorname{cyc}^{m+2 n-x}(T)\right)=\operatorname{comaj}_{\left(1^{m} 2^{n}\right)}(T)+m+2 n-x .
$$


The bottom row of $\operatorname{cyc}^{m+2 n-x}(T)$ is $\left|x^{\prime}\right| z^{\prime} \mid$ where $x^{\prime}=m+2 n$ and $z^{\prime}=m+2 n-x+z$. Then $\operatorname{cyc}^{m+2 n-x+1}(T)$ has bottom row $1 \quad b$ with $b=m+2 n-x+z+1$. The ascent involving $x^{\prime}$ and the entry directly above it in $\operatorname{cyc}^{m+2 n-x}(T)$ is lost when cyc is applied, so

$$
\operatorname{comaj}_{\left(1^{m} 2^{n}\right)}\left(\operatorname{cyc}^{m+2 n-x+1}(T)\right)=\operatorname{comaj}_{\left(1^{m} 2^{n}\right)}\left(\operatorname{cyc}^{m+2 n-x}(T)\right)-(m+n-1)
$$

and thus

$$
\operatorname{comaj}_{\left(1^{m} 2^{n}\right)}\left(\operatorname{cyc}^{m+2 n-x+1}(T)\right)=\operatorname{comaj}_{\left(1^{m} 2^{n}\right)}(T)+n+1-x .
$$

By 5.3 ,

$$
\operatorname{inv}_{\left(1^{m} 2^{n}\right)}\left(\operatorname{cyc}^{m+2 n-x}(T)\right)=\operatorname{inv}_{\left(1^{m} 2^{n}\right)}(T) .
$$

Since $m+2 n$ is in the bottom row of $\operatorname{cyc}^{m+2 n-x}(T)$,

$$
\operatorname{inv}_{\left(1^{m} 2^{n}\right)}\left(\operatorname{cyc}^{m+2 n-x+1}(T)\right)=\operatorname{inv}_{\left(1^{m} 2^{n}\right)}\left(\operatorname{cyc}^{m+2 n-x}(T)\right)-1,
$$

so

$$
\operatorname{inv}_{\left(1^{m} 2^{n}\right)}\left(\operatorname{cyc}^{m+2 n-x+1}(T)\right)=\operatorname{inv}_{\left(1^{m} 2^{n}\right)}(T)-1 .
$$

We can calculate $R_{\left(1^{m} 2^{n}\right), a}$ for $2 \leq a \leq m+2 n$ by the recursion below.

Theorem 6.16 For all $m \geq 1, n \geq 2$, and $2 \leq a \leq m+2 n$,

$$
\begin{aligned}
& R_{\left(1^{m} 2^{n}\right), a}=\sum_{b=2}^{m+2 n-2} \sum_{i=0}^{m+2 n-2-b}\left(t^{i+(n-1) \chi(i<a-b-1)} R_{\left(1^{m} 2^{n-1}\right), b}\right. \\
& \left.+q t^{b-n+i+(n-1) \chi(i<a-2)} R_{\left(1^{m} 2^{n-1}\right), m+2 n-b}\right) .
\end{aligned}
$$

The initial conditions are $R_{\left(1^{m}\right), a}=F_{\left(1^{m}\right)}=[m] !_{t}$ for $m \geq 1$ and $2 \leq a \leq m+1$.

Proof: Let $T \in \mathcal{F}_{\left(1^{m} 2^{n}\right), a}$. Then there exists a unique $U \in \mathcal{F}_{\left(1^{m} 2^{n-1}\right)}$ such that $T=A_{a}\left(\operatorname{cyc}^{i}(U)\right)$ and the bottom row of $U$ is either \begin{tabular}{l|l|l|l|l|l|l|l|} 
& $b$ & or & $b$ & 1
\end{tabular} for some $b$ with $2 \leq b \leq m+2 n-2$ and some $i$ with $0 \leq i \leq m+2 n-2-b$. If the bottom row of $U$ is $1 \quad b$, then by 6.14 and 5.3

$$
\begin{aligned}
\operatorname{comaj}_{\left(1^{m} 2^{n}\right)}(T) & =\operatorname{comaj}_{\left(1^{m} 2^{n}\right)}\left(A_{a}\left(\operatorname{cyc}^{i}(U)\right)\right) \\
& =\operatorname{comaj}_{\left(1^{m} 2^{n-1}\right)}(U)+i+(n-1) \chi(i<a-b-1)
\end{aligned}
$$

and

$$
\operatorname{inv}_{\left(1^{m} 2^{n}\right)}(T)=\operatorname{inv}_{\left(1^{m} 2^{n-1}\right)}(U) .
$$

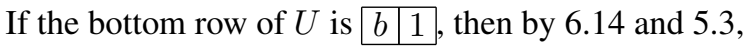

$$
\begin{aligned}
\operatorname{comaj}_{\left(1^{m} 2^{n}\right)}(T) & =\operatorname{comaj}_{\left(1^{m} 2^{n}\right)}\left(A_{a}\left(\operatorname{cyc}^{i}(U)\right)\right) \\
& =\operatorname{comaj}_{\left(1^{m} 2^{n-1}\right)}(U)+i+(n-1) \chi(i<a-2) .
\end{aligned}
$$


Let $U^{\prime}=\operatorname{cyc}^{m+2 n-1-b}(U)$, then, by 6.15 .

$$
\begin{aligned}
\operatorname{comaj}_{\left(1^{m} 2^{n}\right)}(T) & =\operatorname{comaj}_{\left(1^{m} 2^{n-1}\right)}(U)+i+(n-1) \chi(i<a-2) \\
& =\operatorname{comaj}_{\left(1^{m} 2^{n-1}\right)}\left(U^{\prime}\right)+i+b-n+(n-1) \chi(i<a-2)
\end{aligned}
$$

and

$$
\operatorname{inv}_{\left(1^{m} 2^{n}\right)}(T)=\operatorname{inv}_{\left(1^{m} 2^{n-1}\right)}\left(U^{\prime}\right)+1 .
$$

Thus the second term in the recursion accounts for fillings $T$ arising from those $U$ having a decreasing bottom row.

Remark 6.17 We saw earlier that $R_{\left(1^{m} 2^{n+1}\right), 2}(q, t)=F_{\left(1^{m} 2^{n}\right)}(q, t)$ is divisible by $[m] !_{t}[n] !_{t}$. The recursion above easily yields that every $R_{\left(1^{m} 2^{n}\right), a}(q, t)$ is divisible by $[m] !_{t}$. Computations suggest that these polynomials are also divisible by $[n-1] !_{t}$, but this fact does not seem to be evident from the form of our recursion. In particular (in contrast to the situation for two equal columns), we have not given a combinatorial explanation of the divisibility of $F_{\left(1^{m} 2^{n}\right)}(q, t)$ by $[n] !_{t}$.

\section{Fermionic Formula}

In the case of two-column rectangles, we can use our earlier recursion to deduce some fermionic formulas for $F_{\left(2^{n}\right)}(q, t)$ and $R_{\left(2^{n+1}, a\right)}(q, t)$. For each $n \geq 1$, define

$$
P_{n}=\{2,3, \ldots, 2 n\} \times\{2,3, \ldots, 2 n-2\} \times \cdots \times\{2,3,4\} \times\{2\} .
$$

Recall from 6.10 the recursion

$$
R_{\left(2^{n+1}\right), a}=[n]_{t} \sum_{b=2}^{2 n} R_{\left(2^{n}\right), b}\left(t^{(a-b-1)^{+}}+q t^{n-(b+1-a)^{+}}\right), \quad R_{(2), 2}=1 .
$$

Iteration of this recursion immediately yields the following fermionic formula for $R_{\left(2^{n+1}\right), a}$.

Theorem 7.1 For all $n \geq 0$ and $2 \leq a_{0} \leq 2 n+2$,

$$
R_{\left(2^{n+1}\right), a_{0}}=[n] !_{t} \sum_{\left(a_{1}, a_{2}, \ldots, a_{n}\right) \in P_{n}} \prod_{i=1}^{n}\left(t^{\left(a_{i-1}-a_{i}-1\right)^{+}}+q t^{n+1-i-\left(a_{i}+1-a_{i-1}\right)^{+}}\right) .
$$

Now let $\operatorname{PM}\left(K_{2 n}\right)$ denote the set of perfect matchings of the complete graph $K_{2 n}$ on $2 n$ vertices. By definition, an element $M \in \operatorname{PM}\left(K_{2 n}\right)$ is a set partition of $\{1,2, \ldots, 2 n\}$ into $n$ blocks of size 2 . There is a bijection $f: P_{n} \rightarrow \operatorname{PM}\left(K_{2 n}\right)$ that can be defined recursively as follows. For $n=1, f((2))=\{\{1,2\}\}$. Given $n>1$ and $\left(a_{1}, \ldots, a_{n}\right) \in P_{n}$, recursively compute $M^{\prime}=f\left(a_{2}, \ldots, a_{n}\right) \in \operatorname{PM}\left(K_{2 n-2}\right)$. Relabel the perfect matching $M^{\prime}$ to use the vertex set $\{1,2, \ldots, 2 n\} \sim\left\{1, a_{1}\right\}$, and add the edge $\left\{1, a_{1}\right\}$ to obtain the perfect matching $M=f\left(a_{1}, \ldots, a_{n}\right)$. We can use this bijection to change the indexing set for the summation in 7.1 from $P_{n}$ to $\operatorname{PM}\left(K_{2 n}\right)$. We thereby see that $R_{\left(2^{n+1}\right), a}$ can be written in the form

$$
R_{\left(2^{n+1}\right), a}=[n] !_{t} \sum_{M \in \operatorname{PM}\left(K_{2 n}\right)}\left[2^{n}\right]_{M, a}
$$


where $\left[2^{n}\right]_{M, a}$ denotes a $q, t$-analogue of $2^{n}$ that depends on the matching $M$ and the integer $a$. In particular, we have such an expression for $F_{\left(2^{n}\right)}=R_{\left(2^{n+1}\right), 2}$. This yields a $q, t$-analogue of the enumeration formula $\left|\operatorname{PM}\left(K_{2 n}\right)\right|=\frac{(2 n) !}{n ! 2^{n}}$.

It is also natural to incorporate $a$ into the matching $M$ by summing over perfect matchings on $2 n+2$ vertices that contain the edge $\{1, a\}$. This viewpoint produces a formula of the form

$$
R_{\left(2^{n+1}\right), a_{0}}=[n] !_{t} \sum_{\substack{M \in \operatorname{PM}\left(K_{2 n+2}\right): \\\left\{1, a_{0}\right\} \in M}}\left[2^{n}\right]_{M} .
$$

Example 7.2 Suppose $n=3, a_{0}=6$, and

$$
M=\{\{1,6\},\{2,4\},\{3,8\},\{5,7\}\}
$$

We calculate $\left(a_{0}, a_{1}, a_{2}, a_{3}\right)=f^{-1}(M)=(6,3,4,2)$. Referring to 7.1 , we see that

$$
\left[2^{3}\right]_{M}=\left(t^{2}+q t^{3}\right)(1+q)(t+q t) .
$$

\section{Future Work}

The computations in [Nie10] suggest that the recursions considered here may extend to polynomials $F_{\mu}$ indexed by partitions $\mu$ with more than two columns. In particular, the case of a three-column rectangle appears to be tractable, although the required combinatorial manipulations become substantially more involved compared to two-column rectangles. These extensions of the current work, which are treated in the latter part of [Nie10], will be the subject of future papers. 


\section{References}

[Ban07] J. Bandlow. Combinatorics of Macdonald polynomials and extensions. PhD thesis, University of California at San Diego, 2007.

[GH93] A. Garsia and M. Haiman. A graded representation model for Macdonald's polynomials. Proc. Natl. Acad. Sci. USA, 90:3607-3610, 1993.

[GH96] A. Garsia and M. Haiman. A remarkable $q, t$-Catalan sequence and $q$-Lagrange inversion. $J$. Algebraic Combinatorics, 5:191-244, 1996.

[GH98] A. Garsia and M. Haiman. A random $q, t$-hook walk and a sum of Pieri coefficients. $J$. Combin. Theory Ser. A, 82:74-111, 1998.

[GHar] A. Garsia and J. Haglund. A new recursion in the theory of Macdonald polynomials. Annals of Combinatorics, to appear.

[Hag04] J. Haglund. A combinatorial model for the Macdonald polynomials. Proc. Natl. Acad. Sci. USA, 101:16127-16131, 2004.

[Hai01] M. Haiman. Hilbert schemes, polygraphs, and the Macdonald positivity conjecture. J. Amer. Math. Soc., 14:941-1006, 2001.

[Hai02] M. Haiman. Notes on Macdonald polynomials and the geometry of Hilbert schemes. Symmetric Functions 2001: Surveys of Developments and Perspectives, NATO Sci. Ser. II Math. Phys. Chem., 74:1-64, 2002.

[HHL05a] J. Haglund, M. Haiman, and N. Loehr. A combinatorial formula for Macdonald polynomials. J. Amer. Math. Soc., 18:735-761, 2005.

[HHL05b] J. Haglund, M. Haiman, and N. Loehr. Combinatorial theory of Macdonald polynomials I: Proof of Haglund's formula. Proc. Natl. Acad. Sci. USA, 102:2690-2696, 2005.

[Mac88] I. Macdonald. A new class of symmetric functions. Actes du 20e Séminaire Lotharingien, 372/S-20:131-171, 1988.

[Mac95] I. Macdonald. Symmetric functions and Hall polynomials. Oxford University Press, second edition, 1995.

[Nie10] E. Niese. Combinatorial properties of the Hilbert series of Macdonald polynomials. PhD thesis, Virginia Tech, 2010.

[Yoo09] M. Yoo. Combinatorial formula for the Hilbert series of bigraded $S_{n}$-modules. Discrete Math. Theor. Comput. Sci., proc. AK:903-914, 2009. 